\title{
Reflexión de la teoría crítica, la pedagogía revolucionaria y la educación liberadora*
}

\author{
Reflection of the Critical Theory, Revolutionary Pedagogy and Liberating \\ Education
}

\begin{abstract}
José Eriberto Cifuentes Medina
Doctor en Educación. Universidad de Baja California, Tepic, Nayarit, México eribertocifuentes@hotmail.com

Israel Alfonso Moreno Pinzón

Magíster en Administración y Planificación Educativa. Universidad Metropolitana de Educación, Ciencia y Tecnología UMECIT- Panamá. israelmorenopinzon@gmail.com Aura Lucía Camargo Silva

Magíster en Educación. Docente Secretaria de Educación de Boyacá. Colombia. camargoaura@hotmail.com
\end{abstract}

Fecha de recepción: 23 de marzo de 2016

Fecha de aprobación: 17 de mayo de 2017

\section{Cómo citar este artículo/ to reference this article}

Cifuentes Medina, J., Moreno Pinzón, I., \& Camargo Silva, A. (2017). Reflexión de la teoría crítica, la pedagogía revolucionaria y la educación liberadora. Derecho Y Realidad, 15(29): e9084. Recuperado a partir de https://revistas.uptc.edu.co/index.php/derecho_realidad/article/view/9084

Resumen: La investigación realiza un análisis de la teoría crítica desde de Horkheimer, la pedagogía revolucionaria propuesta por Peter McLaren y la educación liberadora en la perspectiva de Paulo Freire, contribuye a desglosar el sentido de la educación en el siglo XX y su proyección en el siglo XXI. Objetivo: Identificar los principales factores de incidencia en la educación y la pedagogía con base en Horkheimer, Peter McLaren y Paulo Freire. Método: El estudio responde a un enfoque cualitativo y que responde a una revisión documental en relación a los factores afines a la época histórica, como los aportes de la Escuela de Frankfurt, la revolución violenta pero también pacífica en diferentes ámbitos de la sociedad mundial y que ha permeado todos los ambientes. Resultados: Se realiza un recorrido histórico y geográfico por Europa, Norteamérica y Suramérica de los tres autores y, se encuentran elementos en común a favor de la pedagogía y la educación, en donde el

\footnotetext{
* Artículo de reflexión. Con algunas modificaciones presentado como ponencia en el "I Congreso Internacional de Antropología Pedagógica, Persona-Educación-Formación en el Contexto Iberoamericano”, 24 al 27 de septiembre de 2014, Bogotá, Colombia.
} 
hombre aparece como el centro del proceso educativo en búsqueda de una revolución sana de la mente y de una educación que lo libere de la esclavitud de la ignorancia.

Palabras clave: teoría crítica, pedagogía revolucionaria, educación liberadora, teoría de la educación, derechos humanos.

\begin{abstract}
The research makes an analysis of critical theory from Horkheimer, the revolutionary pedagogy proposed by Peter McLaren and liberating education in the perspective of Paulo Freire, contributes to breaking down the meaning of education in the twentieth century and its projection in the XXI century. Objective: To identify the main factors of incidence in education and pedagogy based on Horkheimer, Peter McLaren and Paulo Freire. Method: The study responds to a qualitative approach and responds to a documentary review in relation to the factors related to the historical epoch, such as the contributions of the Frankfurt School, the violent but also peaceful revolution in different areas of world society and that has permeated all environments. Results: There is a historical and geographical journey through Europe, North America and South America of the three authors and, there are elements in common in favor of pedagogy and education, where man appears as the center of the educational process in search of a a healthy revolution of the mind and an education that frees it from the bondage of ignorance.
\end{abstract}

Keywords: critical theory, revolutionary pedagogy, liberating education, educational theory, human rights. 


\section{Introducción}

En el recorrido por el camino de la teoría crítica, la pedagogía revolucionara y la educación liberadora se encuentran elementos que permiten entender el engranaje de la educación y la pedagogía en los siglos XX y XXI. La emigración de Adorno a los Estados Unidos y la intensa colaboración que de allí iniciara con Horkheimer pueden ser considerados como la fecha de nacimiento de la teoría crítica, cuyo principal interés sería el mantenerse actual pero también la búsqueda de paradigmas que deberían comprender los planteamientos de una restitución de la filosofía transcendental, y la intención originaria de los exponentes de la teoría crítica fue desarrollar una reflexión filosófica, social y política en articulación con las ciencias sociales.

La pedagogía crítica revolucionaria propuesta por Peter McLaren busca interpretar la práctica pedagógica concreta y hacer de la pedagogía crítica un espacio de reflexión de la teoría y de la práctica. La pedagogía crítica ha comenzado a proporcionar una teoría radical en el análisis de la escuela, pero no es un conjunto homogéneo de ideas que trata de habilitar a los desposeídos y transformar las desigualdades e injusticias sociales existentes, pues lleva a un desarrollo de una pedagogía de revolución desde las escuelas como centro educativo y de transformación humana para luchar por la justicia social y la igualdad social.

La educación liberadora, desde la perspectiva del discurso dialógico de Paulo Freire y Habermas "incluye las dos características del pensamiento y acción critica para el cambio social: a) Defiende clara y radicalmente el objetivo de igualdad; b) apoya decididamente los movimientos sociales igualitarios" (Piérola, 1997, pág. 20). El discurso social que afirma que no podremos desarrollar nuevas teorías y prácticas por el cambio social sin criticar los planteamientos de quienes las critican, y en palabras de Friere: "No es posible estar a favor de alguien sin estar contra alguien, que está contra ese de quien yo estoy a favor" (Piérola, 1997, pág. 30).

\section{Consideraciones teóricas}

En el desarrollo teórico de la presente investigación se acude de manera breve a varios tópicos de profundización: generalidades de pedagogía, teoría crítica de Max Horkheimer, pedagogía revolucionaria de Peter McLaren y educación liberadora de Paulo Freire; en donde se entrelaza el desarrollo de la filosofía, la pedagogía y la educación desde la perspectiva de tres pensadores en la historia de la humanidad. 


\subsection{Generalidades de pedagogía}

La pedagogía, en una reflexión inicial, puede considerarse como una disciplina enfocada en el estudio del fenómeno educativo, desde la perspectiva filosófica, científica y técnica, como se refiere en la Tabla 1.

Tabla 1. Perspectivas de la pedagogía

\begin{tabular}{|c|l|}
\hline PERSPECTIVA & \multicolumn{1}{c|}{ BREVE DESCRPCIÓN } \\
\hline FILOSÓFICA & $\begin{array}{l}\text { Se ocupa de determinar cómo debería llevarse a cabo el proceso educativo. Trata } \\
\text { la totalidad del fenómeno señalado, explicándolo y consignando cuáles deberían } \\
\text { ser los fines para los que educamos, atendiendo al tipo de hombre y de sociedad } \\
\text { que se piensa formar. Esta perspectiva señala el deber ser de la educación. }\end{array}$ \\
\hline CIENTÍFICA & $\begin{array}{l}\text { Se acude a la pedagogía como disciplina científica, en donde se pueden advertir } \\
\text { dos niveles: el primero es aquel en que la pedagogía se aboca a la búsqueda de las } \\
\text { leyes que expliquen el fenómeno educativo, a efectos de exponerlo en una teoría, } \\
\text { así tenemos a la pedagogía como ciencia pura. Y el segundo se puede distinguir } \\
\text { cunado la pedagogía se inclina por el estudio de los problemas de la educación y } \\
\text { sus posibles alternativas de solución, atendiendo al conocimiento que se tiene del } \\
\text { fenómeno; desde este ángulo interpretamos a la pedagogía como una ciencia } \\
\text { aplicada. }\end{array}$ \\
\hline TÉCNICA & $\begin{array}{l}\text { Cuando se trata de aplicar herramientas e instrumentos en la resolución de } \\
\text { problemas, la pedagogía se convierte en técnica. }\end{array}$ \\
\hline
\end{tabular}

Fuente: De La Torre (2005)

Se puede afirmar que la pedagogía es la noción más general que comprende a la didáctica y a la educación, mientras que la didáctica es más particular y hace referencia a un contexto específico. Además, el debate epistemológico avanza en la hermenéutica de dos formas de la pedagogía: tradicional y dialogante.

Tabla 2. Pedagogía tradicional y pedagogía dialogante

\begin{tabular}{|c|l|}
\hline PEDADOGÍA & \multicolumn{1}{c|}{ BREVE DESCRPCIÓN } \\
\hline \multirow{5}{*}{ TRADICIONAL } & $\begin{array}{l}\text { Ha dominado la mayor parte de instituciones educativas a lo largo de la historia } \\
\text { humana y en la mayoría de regiones del mundo; aun así, solo ha recibido unas } \\
\text { pocas líneas de sustentación teórica. En la pedagogía tradicional, el maestro es el } \\
\text { transmisor de los conocimientos y las normas culturalmente construidas y aspira a } \\
\text { que, gracias a la función, dichas informaciones y normas estén al alcance de las } \\
\text { nuevas generaciones. El maestro "dicta la lección" a un alumno que recibirá las } \\
\text { informaciones y las normas transmitidas para aprenderlas e incorporarlas entre } \\
\text { sus saberes. El niño obtiene del exterior el conocimiento y las normas que la } \\
\text { cultura construyó y gracias a ellos se convierte en hombre. }\end{array}$ \\
\hline \multirow{5}{*}{ DIALOGANTE } & $\begin{array}{l}\text { Reconoce que el conocimiento se construye por fuera de la escuela, pero que es } \\
\text { reconstruido de manera activa e interestructurada a partir del diálogo pedagógico } \\
\text { entre el estudiante, el saber y el docente y que, para que ello se presente, es } \\
\text { condición indispensable contar con la mediación adecuada de un maestro, que } \\
\text { favorezca de manera intencionada, mediada y transcendente el desarrollo integral } \\
\text { del estudiante. Un modelo que concluya que la finalidad de la educación no puede } \\
\text { estar centrada en el aprendizaje, como desde hace siglos ha creído la escuela, sino } \\
\text { en el desarrollo. }\end{array}$ \\
\hline
\end{tabular}

Fuente: De Zubiría (2006)

En síntesis dialéctica tendría que recuperar de la escuela activa y de los enfoques constructivistas el reconocimiento del papel activo que cumple el estudiante en todo proceso de aprendizaje y la finalidad de la comprensión y el desarrollo intelectual que le 
asignan a la escuela. La hermenéutica de la pedagogía tradicional no es una cuestión totalmente obsoleta o pasada a la historia, pues en su momento fue un hito y ahora le corresponde a la historia valorar sus aportes y su significado transcendente, al igual que punto de partida para otras pedagogías, como la dialogante, entre otras; todas preocupadas por el estudio del fenómeno educativo y su propósito de transformación de la dinámica entre el docente y el estudiante en la construcción de conocimiento.

El análisis sistemático de la pedagogía en la presente disertación permite, además, realizar un análisis sucinto de la teoría crítica de Max Horkheimer, de la pedagogía revolucionaria de Peter McLaren y la educación liberadora de Paulo Freire, para proyectar una reflexión a la vanguardia del siglo XX para la comprensión actualizada del fenómeno educativo.

\subsection{Teoría crítica desde Horkheimer}

El estudio de los fundamentos de la teoría crítica de Horkheimer, que están vinculados con las postulaciones de la Escuela de Frankfurt, pasando por la propuesta de pedagogía de la revolución de McLaren, hasta llegar a formar parte del proyecto educativo que propone el pedagogo Freire en América Latina, implican un recorrido contemporáneo por el siglo XXI.

De esta manera, Horkheimer, como miembro de la Escuela de Frankfurt, desde la investigación social y la perspectiva de la teoría crítica:

[...] convirtió así directamente en crítica de la sociedad, sin renunciar a la pretensión de ser filosofía. Y así, muchos de los malentendidos y falsos juicios con respecto a las intenciones de la Teoría Crítica tienen su causa en la incapacidad de los intérpretes de tomar en cuenta ambas cosas al mismo tiempo. (Geyer, 1985, pág. 80)

La teoría crítica, desde la perspectiva de Horkheimer, que se da en la formación académica que él recibió y que se cultiva en el seno de la Escuela de Frankfurt y que funda "los cimientos de una sociedad racional de Horkheimer, parte de la premisa de hacer de la filosofía social como una opción por la Teoría Crítica" (Suárez, 2009, pág. 145), mientras se desarrolla un diálogo del contexto social entre la filosofía como madre de todas las ciencias y las categorías de las ciencias sociales desde la perspectiva del materialismo. Y

Dentro del contexto filosófico contemporáneo hablar de la Teoría Crítica de la sociedad o de la Escuela de Frankfurt, se enfoca directamente el programa de reflexión filosófica, investigación social y orientación política asociada a nombres como Theodor Adorno, Max Horkheimer, Walter Benjamín, Herbert Marcuse, Habermas y actualmente Axel Honneth. (Suárez, 2009, pág. 147)

Continuando con la perspectiva de Suárez (2009), este autor se refiere a Horkheimer como uno de los exponentes de la teoría crítica, quien plantea desarrollar una reflexión filosófica, social y política que pueda articularse con las ciencias sociales, cuyos propósitos se centran en la investigación social con raíces teóricas de la filosofía y el andamiaje práctico de las ciencias sociales, preservando el imaginario de la interdisciplinariedad. 
El estudio de la teoría crítica también se da en medio de fenómenos históricos, teniendo en cuenta las circunstancias de Alemania en el siglo XX, como la emigración, el nacionalismo alemán, Auschwitz, los movimientos estudiantiles, el positivismo, el racionalismo crítico, entre otras; cuyo propósito principal es la reflexión filosófica desde lo social. En 1923 se fundó el Instituto de Investigación Social de Frankfurt, y en su inauguración en 1924, como director del Instituto, Carl Grünberg, "profesor de derecho y ciencias políticas de la Universidad de Viena presentó el programa de trabajo futuro orientado hacia el marxismo en sentido científico pero no político-partidista [...] en 1930 Horkheimer ocupó el cargo como director del Instituto como sucesor de Grünberg” (Friedrich, 1982, pág. 50).

Los intereses de Horkheimer y los demás miembros del Instituto apuntaban a vincular recíprocamente la filosofía y el análisis de la sociedad, "Al mismo tiempo, esta vinculación era entendida como la investigación de posibilidades de una modificación del orden social a través de una praxis humana" (Friedrich, 1982, p. 8). Predomina la amistad de Horkheimer con Adorno y este último asume la codirección del Instituto en 1945 a pesar de que en 1932 estuvo en peligro cuando el director del Instituto y Pollock se negaran a propiciar una habilitación de Walter Benjamín.

\section{Los trabajos de}

Horkheimer sobre la fundamentación de una teoría critica de la historia y la sociedad puede ser considerada como elementos de una futura filosofía de la historia en la medida en que ellos derivan el momento dinámico constitutivo de la teoría que había de concebir [...] (Friedrich, 1982, pág. 60).

Porque, de alguna manera, la teoría crítica ha subrayado siempre su carácter históricosituacional, colocándolo en el primer plano de sus análisis, naturalmente sin la unilateralidad e impertinencia de lo meramente biográfico.

La teoría crítica;

Es solo comprensible a partir de la situación de los años veinte de nuestro siglo y de las intensas discusiones que determinaron el clima espiritual de aquella época. La Revolución Rusa de octubre, el establecimiento de sistemas democráticos en las monarquías decadentes del Centro de Europa, la progresiva industrialización [...]. (Friedrich, 1982, pág. 70)

Ante la decadencia de las diversas tradiciones, se considera al marxismo como una variable histórica-dialéctica de materialismo en los espacios de los universitarios, quienes hasta el momento se consideraban defensores de los valores tradicionales.

Max Horkheimer, en su tiempo de exilio americano, planteó tres problemas fundamentales: "en primer lugar se interesó, por las causas que condujeron hacia el triunfo del fascismo en Europa, en segundo lugar examinó el desarrollo propio del contexto americano y en tercer lugar, revisó la base marxista de la teoría crítica" (Suárez, 2009, pág. 148) Se sintetiza su pensamiento en el periodo del exilio así: "Lo que nos habíamos propuesto era nada menos que comprender por qué la humanidad, en lugar de entrar en un estado verdaderamente humano, se hunde en un nuevo género de barbarie" (Suárez, 2009, pág. 148). De manera que la teoría crítica parte de la premisa de hacer filosofía social y desarrollar una 
interpretación dialéctica de lo social entre la filosofía y las teorías de las ciencias sociales y desde la perspectiva del materialismo interdisciplinario.

\subsection{De la teoría crítica a la pedagogía de la revolución de Peter McLaren}

Por su parte, la propuesta de McLaren se puede denominar como una pedagogía crítica para la transformación, dada más a la producción de conocimiento, desde la perspectiva de la revolución, sin que se silencie la voz de los estudiantes para una construcción social óptima:

McLaren presenta su visión más radicalmente marxista, teóricamente elaborada y poéticamente articulada de una educación por la justicia social y el cambio que desarrolló desde que se convirtió en uno de los más destacados representantes de la pedagogía crítica en América del Norte, a mediados de los ochenta. (Huertas-Charles, 2007, pág. 80)

McLaren hace grandes aportes para el desarrollo de la sociedad en el mundo contemporáneo de la educación, y en su reflexión en contra del capitalismo se centra en una filosofía marxista humanista en medio de un estilo original. Vale citar un ejemplo en donde McLaren realiza un trabajo "en dos contextos distintos: por un lado, las áreas rurales del estado de Río de Janeiro, donde trabajó con maestros de escuelas de primarias y secundarias y por otro, un área metropolitana [...] en clases de maestrías" (Huertas-Charles, 2007, pág. 90). Por ello, el texto fundamental sobre pedagogía de McLaren se denomina: "La vida en las escuelas".

El largo viaje de McLaren hacia el marxismo comenzó atravesando el vasto y poco explorado territorio de las teorías posmodernas y posestructuralistas. Tanto en Pedagogía crítica y cultura depredadora (1995) como en el multiculturalismo revolucionarios (1997), McLaren experimentó, flirteó incluso, con los abordajes posmodernos y posestructuralistas de la teoría y la práctica educativa. Exploró y analizó desfachatadamente las tensiones y debates por largo tiempo existentes entre los abordajes culturalista y materialista de la educación, así como los apremiantes debates filosóficos respecto de la relación entre estructura y capacidad de actuar. (Huertas-Charles, 2007, pág. 75)

A McLaren se le puede considerar como un pedagogo del mundo contemporáneo, con aportes brillantes a la educación, la pedagogía y la sociedad,

Frente a las teorías posmodernas que el fin de la clase trabajadora, en las que según McLaren analiza la globalización como una forma de imperialismo, con una perspectiva crítica que se fundamenta en la teoría marxista y en el concepto de clase social. (Aguirre, 2003, pág. 26)

Se puede ver que está de acuerdo en que la pedagogía ha de ser crítica, cuyo papel será el de contribuir a la creación de una sociedad equitativa, justa, sostenida por los valores de la cooperación, la solidaridad y todos aquellos que le asistan por el derecho de ser humana.

Para McLaren, la educación se ha de fundamentar en ser revolucionaria crítica, que docentes y estudiantes puedan ser agentes para la trasformación de la sociedad sumergida en la globalización y el capitalismo, "una pedagogía capaz de comprometer cada día de la 
vida como vivida en medio de una tendencia global capitalista conducente hacia un imperio, una pedagogía que hemos denominado revolucionaria crítica" (Aguirre, 2003, pág. 14)

En la perspectiva de McLaren, la pedagogía:

crítica evita todo enfoque de la pedagogía que la redujera a la enseñanza de habilidades de miradas estrechas y aisladas de los debates y contexto de la discusión en los que se las utiliza. La pedagogía crítica ha sido esterilizada, vulgarizada, domesticada y purgada de su profundidad teórica y descernimiento. (McLaren, 2005, pág. 42)

La pedagogía crítica "resuena con la sensibilidad del símbolo hebreo tikum, que significa: curar, reparar y transformar el mundo; todo lo demás es comentario [...] la pedagogía crítica es tan revolucionaria como los primeros propósitos de los autores de la declaración de la independencia" (McLaren, 2005, p. 256). Cuenta con principios como la política, la cultura, la economía, entre otros; en donde sobresale la "convicción de que para la escuela es una prioridad ética dar a conocer al sujeto y a la sociedad sobre el dominio de habilidades técnicas, que están primordialmente atadas a la lógica del mercado de trabajo" (McLaren, 2005, pág. 48).

\section{La importancia de la pedagogía crítica se entrelaza con}

los teóricos críticos que comienzan con la premisa de que los hombres y las mujeres no son en esencia libres y que habitan un mundo repleto de contradicciones y asimetrías de poder y privilegios. El educador crítico aprueba teorías que son, ante todo dialécticas; esto es, teorías que reconocen los problemas de la sociedad como algo más que simples hechos aislados de los individuos o deficiencias en la estructura social. (McLaren, 2005, pág. 56)

Para comprender el desarrollo de la pedagogía revolucionaria es necesario revisar "el trabajo alternativo de McLaren -tal como Cries from the corridor (1980), La escuela como un performance ritual (1986), la vida en las escuela (1989) y Pedagogía crítica y cultura depredadora (1995, tremendamente refrescantes e invaluables)" (Huerta-Charles, 2007, pág. 85). Son obras que contribuyen a interpretar la educación multicultural y la pedagogía crítica y de la revolución en el contexto educativo.

La teoría crítica a la pedagogía de la revolución busca revelar el hecho de que las prácticas educativas y el desarrollo del conocimiento siempre se producen dentro de un ámbito con condiciones sociales e históricas particulares y, desde luego, cualquier producción va acompañada de una investigación de relación entre la ideología y el poder según el contexto. El pensamiento de McLaren continúa iluminando el desarrollo de la presente investigación,

[...] en tanto la lógica del capitalismo transnacional ha regido la política y práctica educativa, en el trabajo de McLaren había y sigue habiendo en libros tales como multiculturalismo revolucionario (1997) y EL Che Guevara, Paula Freire y la pedagogía de la revolución (2000) una extraña sinceridad y coraje; en donde, términos tales como opresión, explotación capitalista y liberación no quedan envueltos en un lenguaje 
domesticado que purga en silencio el dolor que esas mismas palabras intentar nombrar. (Huerta-Charles, 2007, pág. 65)

Siguiendo en el proceso de interpretación y comprensión del pensamiento de McLaren respecto del multiculturalismo que se desarrolla en cuatro fases: conservador, liberal, liberal de izquierda y multiculturalismo crítico y de resistencia:

[...] el primero, debido a que su orientación hacia las diferencias culturales dentro del aula está enteramente basada en el modelo del déficit. El segundo, dando por sentado que todos los seres humanos son iguales y deberían competir en un campo de juego equilibrado dentro de la sociedad capitalista. El tercero, de ellos, por revalorizar las diferencias culturales y la autenticada desde el relativismo cultural y el esencialismo, la cuarta categoría de McLaren el multiculturalismo crítico y de resistencia- [...]. (Huerta-Charles, 2007, pág. 58)

Para él, la cultura es entendida como la

Encarnación de las experiencias y comportamientos que son el resultado de la desigual distribución de poder sobre la base de lineamientos tales como raza, genero, sexualidad y aptitudes. Esto quiere decir que a la cultura la conforman las experiencias de vida y las formas institucionales organizadas en torno de distintos elementos de lucha y dominio. (Huerta-Charles, 2007, pág. 67)

En el ambiente educativo es necesario comprender el multiculturalismo, pues en realidad no se trata de que los docentes desarraiguen a los estudiantes de sus tradiciones culturales, políticas, religiosas, etc., sino que se trata de un intercambio de ideas más crítico y democrático, en donde la posición, las experiencias y las percepciones de cada uno de los involucrados se conviertan en el punto de partida para el diálogo y un texto de debate académico y de construcción de conocimiento y de la transformación social de cada sujeto.

De este modo, McLaren se considera insigne entre los pedagogos de América por su planteamiento en relación con una educación liberadora del ser humano, como se evidencia en sus diversas obras, en donde se discute la injusticia social y que esta puede ser contrarrestada por la educación liberadora; en su lucha por los desamparados, los pobres y los oprimidos, la educación es la salida de la opresión en donde unos pocos se creen superiores a los demás.

\subsection{Educación liberadora desde el contexto latinoamericano}

El contexto latinoamericano en el siglo XX está enmarcado en una serie de acontecimientos que le son propios (teología de la liberación) y de otros que han sucedido en diversas partes del planeta (Primera y Segunda Guerra Mundial) y que han terminado por afectar con un coletazo la situación de América Latina. Por mencionar unos pocos acontecimientos que con muchos más, han hecho que en el pasado siglo y en los comienzos del XXI, en todas dimensiones y direcciones, se pueda progresar (en aspectos tecnológicos, etc.) y tal vez en otros, retroceder (como en el cuidado de la ecología, entre otros), en medio de la compleja realidad donde la educación y sus actores tienen un gran papel que, por cierto, ha de ser revolucionario-crítico, según Peter McLaren, y liberador, según Paulo Freire. 


\section{Entonces, seguir a Paulo Freire}

[...] en su obra más revolucionaria, La Pedagogía del Oprimido, demanda un examen crítico de la realidad, una definición de categorías de análisis y una postura trasparente para observar la relación entre oprimidos y opresores. Con los primeros, manifestados en los excluidos y en los más pobres, se desarrolla una propuesta libradora que trasciende el iluminismo mediante las posibilidades de la acción y del compromiso [...]. (Aguirre, 2003, pág. 20)

Ahora bien, los propósitos de Freire nacen de las necesidades que se presentan en el entorno social, que motivan a los agentes de la educación, principalmente a los estudiantes, a revisar la concepción de la indagación y la reflexión continua con el fin de transformar la realidad social con la teoría y la práctica. El reto de los docentes es grande, pues Freire decía en sus máximas: "enseñar exige respeto a los saberes de los educandos, enseñar exige las corporización de las palabras por el ejemplo, enseñar exige seguridad, capacidad profesional y generosidad y enseñar exige saber escuchar" (Aguirre, 2003, pág. 25)

De esta forma, Freire promueve la necesidad de desarrollar la pedagogía de la pregunta, siempre se está escuchando la pedagogía de la respuesta y más cuando los docentes responden a preguntas que los estudiantes no han formulado, impulsar los principios de una alfabetización más allá de enseñar el abecedario. En este medio,

cuando el maestro sabe su saber y con él piensa el mundo, puede formar en sus alumnos la capacidad crítica, esto es, la libertad de pensar, de elegir y construir las ideas propias para reconocer cuando algo es bueno o malo, cierto o falso; capacidad que requiere ser expresada mediante una acción que produzca transformaciones. Lo cual, a todas luces, para él, iba en contra de las posibilidades de la educación vigente que silenciaba y homogeneizaba para la reproducción de la pasividad social. (Echeverry, 2007, pág. 14)

La propuesta de Freire frente a la educación ha de ser aquella que libere al ser humano "de componentes alienantes: fuerza posibilitadora del cambio y constructora de libertad, para la sociedad es indispensable, a través de la educación, alcanzar la autoconciencia que haga posible la reflexión sobre su tiempo y su espacio en clave de los oprimidos" (Echeverry, 2007, pág. 16) En definitiva, la educación ha de liberar al ser humano y no domesticarlo, es decir, la educación no debe estar alienada y no debe ser alienante, antes bien, debe ser de cambio, de transformación total de la sociedad.

Se entrelaza la propuesta de Illich y Freire en donde se evidencia que "compartían los fundamentos humanistas cristianos de su sufrimiento por la sociedad enajenada. Fue Paulo Freire quien aportó la crítica que más rédito ha dejado, con vistas a producir alternativas al modelo de vinculo pedagógico instalado por la modernidad latinoamericana" (Puiggrós, 2005, pág. 67). Su inminente denuncia constituyó una ruptura epistemológica con la representación del sujeto pedagógico que contiene el sistema educativo moderno.

El modelo tradicional que se apoya

en la unicidad del saber y educador e identifica al educando con la famosa tabula rasa. Freire defiende el quehacer de uno y de otro como funciones impersonales, lo cual habilita el intercambio cultural entre el campesino brasileño y el maestro. (Puiggrós, 2005, pág. 76) 
Se le otorga la importancia de los saberes adquiridos por los estudiantes, ya que no solo es el profesor el dueño y amo del saber y el conocimiento, pues el educando ha adquirido algunas cosas en su medio cultural, y la educación ya no es imposición de saberes, sino que implica comprender que enseñando y aprendiendo pueden adquirirse conocimientos y saberes que permiten imaginar, soñar y construir futuros.

El recorrido se inicia en Europa (Alemania) con Max Horkheimer: teoría crítica e investigación social (Escuela de Frankfurt), pasando por Norteamérica (Canadá) desde la perspectiva de Peter McLaren: pedagogía revolucionaria crítica, y termina en América Latina (Brasil) con uno de los pedagogos más destacados: Paulo Freire, en quien se entrelaza la educación liberadora y la pedagogía del oprimido, con la perspectiva de una educación que libere al ser humano en pro de un desarrollo social justo, solidario con valores de progreso social equilibrado, en donde el hombre le importe a sí mismo y a los demás, desde la esencia de su antropología como estudio de sí y su proceso de educabilidad.

Paulo Freire se puede considerar como "uno de los mayores y más representativos educadores del siglo XX. Su pedagogía muestra un nuevo camino para la relación entre educadores y educandos, camino éste que consolidad una propuesta político-pedagógica definiendo educador y educando como sujetos del proceso de construcción del conocimiento" (Ossa, 2007, pág. 61), mediatizados por el mundo, apuntando a la transformación social y a la construcción de una sociedad justa, democrática e igualitaria.

En el presente escrito se aclara que Freire jamás atacó la escuela, "sino que dirigió su crítica a la relación pedagógica entre educadores y educandos de una manera más general, es decir, con categorías aplicables no solamente a docentes y a los alumnos escolares sino a todos los sujetos sociales vinculados mediante la educación” (Ossa, 2007, pág. 62).

Se considera que para Freire es importante enseñar y, por ende, se ha de valorar al docente en el desarrollo de la construcción de conocimiento con el educando y todo este engranaje, para entender la educación de una manera diferente; todo lo cual es entendido como el movimiento de la "pedagogía de la liberación", que propicia un panorama crítico y propositivo para el desarrollo y transformación del sistema educativo moderno.

\section{Conclusiones}

El análisis de la teoría crítica de Max Horkheimer ha permitido comprender el desarrollo epistemológico, la importancia del Instituto de Frankfurt y el desarrollo histórico del mismo, al igual que la interpretación coherente de la teórica crítica desde la relación de la filosofía social con la teoría de las ciencias sociales.

La pedagogía crítica y revolucionaria desafía el supuesto de que las escuelas operan como espacios mayores de movilidad social y económica, pues estos espacios están allí para promover la igualdad y brindar la oportunidad a muchos grupos de estudiantes de convertirse en ciudadanos críticos y activos en pro de una sociedad justa e igualitaria. Por eso, los maestros de la tradición crítica sostienen que la corriente educativa dominante 
mantiene una tendencia injusta que da como resultado la trasmisión y la reproducción de la cultura.

En los contextos educativos

tenemos motivos para ser críticos y proponer un cambio que mejore nuestra educación y nuestra sociedad. La revolución de la información posibilita realizar muchos de nuestros sueños igualitarios pero, sin embargo, el modelo selectivo que actualmente la preside está incrementando las desigualdades. (Piérola, 1997, pág. 84)

En el ambiente educativo, los profesores son los principales protagonistas para promover en ellos mismos y en sus educandos un espíritu crítico revolucionario, en la búsqueda de la transformación de una sociedad justa y la invitación a la no-violencia pero no al pacifismoconformista, sino al desarrollo humano integral equilibrado para el progreso y el mutuo entendimiento de todos.

En la perspectiva de socialización y educación en la época postmoderna, la escuela cumple tres aspectos fundamentales:

los procesos de socialización o función socializada, la función política y la función educativa de la escuela. Se ha de entender a la escuela como institución social en la que se encuentran grupos de individuos que viven en entornos sociales más amplios, ejerce poderosos influjos de socialización. (Piérola, 1997, pág. 92)

Desde allí se dan los intercambios humanos, comienza el desarrollo del contexto político y económico y, en definitiva, se construye ambiente de cultura, se fortalece la propia y se va transformando la actual; por ello se comprende el movimiento de Freire como una revolución del pensamiento pedagógico universal.

\section{Referencias}

Aguirre, M. L. (2002-2003). La globalización del capital, la pedagogía crítica y las consecuencias del 11 de septiembre: entrevista con Peter McLaren, segunda parte. Revista Opciones Pedagógicas, (26 y 27), 13-49.

De La Torre, F. (2005). 12 Lecciones de pedagogía, educación y didáctica. México: Alfa Omega.

De Zubiría J. (2006). Los modelos pedagógicos. Hacia una pedagogía dialogante. Bogotá: Magisterio.

Echeverry, J. C. (2007). Aproximación a Paulo Freire en clave de hoy. Revista UniPluri/versidad, 7(2), 13-23.

Freire, P. (2005). Cartas a quien pretende enseñar. Buenos Aires: Siglo XXI. 
Friedrich, G. C. (1982). Teoría crítica: Max Horkheimer, Theodor W. Adorno. (1. ${ }^{\mathrm{a}}$ ed.). Barcelona: Laia.

Geyer, C. F. (1985). Teoría crítica de Max Horkheimer y Theodor W. Adorno. Barcelona: Alfa.

Huertas-Charles, L. (2007). De la pedagogía critica a la pedagogía de la revolución. Ensayos para comprender a Peter McLaren. México: Siglo XXI.

McLaren, P. (2005). La vida en las escuelas: una introducción a la pedagogía en los fundamentos de la educación. (4. ${ }^{\text {a }}$ ed.). Buenos Aires: Siglo XXI Editores S.A.

Ossa, J. (2007). Paulo Reglus Freire Neves. Revista Uni-Pluri/versidad, 7(2), 61-62.

Piérola, J. et al. (Coords.). (1997). Ensayos de pedagogía crítica. (1. ${ }^{\text {a }}$ ed.). Madrid: Editorial Popular.

Puiggrós, A. (2005). De Simón Rodríguez a Paulo Freire. Educación para la integración iberoamericana. (1. ${ }^{a}$ ed.). Bogotá: Unidad Editorial, Edición del Convenio Andrés Bello.

Suárez, J. R. (2009, dic.). Compasión y solidaridad política, sentimientos morales propios para superar una época en estado de indigencia: perspectiva vista desde Max Horkheimer. Revista Eidos de Filosofía, 11, 144-149.

\section{Derechos de autor}

Los derechos de autor de este artículo son retenidos por los autores, con los derechos de primera publicación otorgados a la revista. Este es un artículo de acceso abierto distribuido bajo los términos y condiciones de la licencia Creative Commons: Reconocimiento-NoComercial-CompartirIgual 4.0 $\underline{\text { Internacional }}$ 